\title{
Ring-diagram summations and the self-energy of the uniform electron gas at its weak-correlation limit
}

\author{
J. Cioslowski ${ }^{a, b)}$ AND P. Ziesche ${ }^{a)}$ \\ ${ }^{a)}$ Max-Planck-Institut für Physik komplexer Systeme, Nöthnitzer Str. 38, D-01187 \\ Dresden, Germany \\ b) Institute of Physics, University of Szczecin, Wielkopolska 15, 70-451 Szczecin, Poland
}

PACS 71.10.Ca, 05.30.Fk

\begin{abstract}
Ring-diagram summations (equivalent to the random-phase approximation) for several properties of the homogeneous electron gas, such as the total energy components $t$ and $v$, the chemical potential $\mu$, and the quasi-particle weight $z_{\mathrm{F}}$, are reexamined. The ring-diagram summations of the self-energy $\Sigma(k, \omega)$ that yield the correct small$r_{s}$ asymptotics of $v, \mu$, and $z_{\mathrm{F}}$ are identified with the help of rigorous theorems of Galitskii-Migdal, Hugenholtz-van Hove, and Luttinger-Ward. The lowest-order approximation to the self-energy is given by the product of the non-interacting Green's function $G_{0}$ and the static bare Coulomb repulsion $v_{0}$, whereas replacing $v_{0}$ by the ring-diagram-summed dynamically screened interaction $v_{\mathrm{r}}$ yields the proper lowestorder correction to $\Sigma(k, \omega)$. The alternative replacement of $G_{0}$ by the ring-diagramsummed $G_{\mathrm{r}}$ contributes only to the higher-order terms, providing measures of the correlation strength.
\end{abstract}

Typeset by REVTEX 


\section{INTRODUCTION}

Although being an artificial construct, the homogeneous electron gas (HEG) constitutes an important model system for electronic structure theory [1]. The ground state of spinunpolarized HEG is characterized by only one parameter, namely the radius $r_{s}$ of the WignerSeitz sphere that contains one electron on average [2]. It determines the Fermi wave number as $k_{\mathrm{F}}=1 /\left(\alpha r_{s}\right)$ [where $\alpha=(4 /(9 \pi))^{1 / 3}$, and measures simultaneously the interaction strength and the particle density; high density corresponding to weak interaction and hence weak correlation (for recent papers on this limit see refs. [3]-[6]). One could naively expect that at this weak-correlation limit the bare Coulomb repulsion $v_{0}(q)=\alpha r_{s} / q^{2}$ (where momenta and energies are measured in units of $k_{\mathrm{F}}$ and $k_{\mathrm{F}}^{2}$, respectively) can be treated as perturbation. However, already in his early work on the HEG, Heisenberg [7] has shown that ordinary perturbation theory fails in this case. With $e_{0}=3 / 10$ being the energy per particle of the ideal Fermi gas and $e_{\mathrm{x}}=-\frac{3}{4} \frac{\alpha r_{s}}{\pi}$ being the exchange energy in the lowest (first) order, the total energy $e=e_{0}+e_{\mathrm{x}}+e_{\mathrm{c}}$ defines the correlation energy $e_{\mathrm{c}}=e_{2}+e_{3}+\cdots$, where $e_{n} \sim\left(\alpha r_{s}\right)^{n}$ [note that $\tilde{e}=k_{\mathrm{F}}^{2} e=e /\left(\alpha r_{s}\right)^{2}$ gives the energy in atomic units]. In the second order, there is a direct term $e_{2 \mathrm{~d}}$ and an exchange term $e_{2 \mathrm{x}}$ so that $e_{2}=e_{2 \mathrm{~d}}+e_{2 \mathrm{x}}$. The direct term $e_{2 \mathrm{~d}}$ diverges logarithmically along the Fermi surface (i.e. for the vanishing transition momenta $\left.q \rightarrow 0, e_{2 \mathrm{~d}} \rightarrow \ln q\right)$. This failure of perturbation theory has been remedied by Macke [8] with an appropriate partial summation of higher-order terms up to an infinite order that describes screening effects and the collective mode plasmon with a cut-off momentum $q_{c}=\sqrt{4 \alpha r_{s} / \pi}$. This ring-diagram summation, which is equivalent to the random-phase approximation (RPA), yields $e_{\mathrm{c}}=\left(\alpha r_{s}\right)^{2}\left(a \ln r_{s}+\right.$ const $\left.+\cdots\right)$, where $a=(1-\ln 2) / \pi^{2} \approx 0.031091$, for the correlation energy at the weak-correlation limit. This result has been subsequently confirmed by Gell-Mann and Brueckner [9]. The logarithmic behavior of $e_{\mathrm{c}}$ at the weak-correlation limit carries over to its kinetic and potential components through the virial theorem [10]

$$
\begin{array}{r}
t_{\mathrm{c}}=-r_{s}^{2} \frac{d}{d r_{s}} \frac{1}{r_{s}} e_{\mathrm{c}}=-\left(\alpha r_{s}\right)^{2}\left(a \ln r_{s}+\mathrm{const}+\cdots\right), \\
v_{\mathrm{c}}=r_{s} \frac{d}{d r_{s}} e_{\mathrm{c}}=\left(\alpha r_{s}\right)^{2}\left(2 a \ln r_{s}+\mathrm{const}+\cdots\right) .
\end{array}
$$

Note that $t_{0}=e_{0}, t_{\mathrm{x}}=0, v_{\mathrm{x}}=e_{\mathrm{x}}$, and $e_{\mathrm{c}}=t_{\mathrm{c}}+v_{\mathrm{c}}$. It has been shown [5] that these small- $r_{s}$ non-analyticities result from the ring-diagram summation for the momentum distribution 
$n(k)[11]$ and for the static structure factor $S(q)$ [13]. In the lowest order, $n(k)$ diverges along the Fermi surface, $n(k \rightarrow 1) \sim \mp 1 /(k-1)^{2}$ for $k>1$, and $S(q)$ diverges for $q \rightarrow 0$. This makes $t_{2 \mathrm{~d}}$ and $v_{2 \mathrm{~d}}$ diverge correspondingly. The ring-diagram summations remove this unphysical behavior $[5,11,13]$. The chemical potential $\mu=\mu_{0}+\mu_{\mathrm{x}}+\mu_{\mathrm{c}}$, where $\mu_{0}=1 / 2$ and $\mu_{\mathrm{x}}=-\alpha r_{s} / \pi$, enters our considerations through the Seitz theorem [14] ,

$$
\mu_{\mathrm{c}}=\left(\frac{5}{3}-\frac{1}{3} r_{s} \frac{d}{d r_{s}}\right) e_{c}=\left(\alpha r_{s}\right)^{2}\left(a \ln r_{s}+\text { const }+\cdots\right) .
$$

In the following, we use the term "small- $r_{s}$ " with the meaning "RPA in the lowest order", i.e. we derive and discuss here only the terms containing $\ln r_{s}$ or those related to them.

The self-energy $\Sigma(k, \omega)$ is defined by

$$
G=G_{0}+G_{0} \Sigma G, \quad G_{0}(k, \omega)=\frac{\Theta(1-k)}{\omega-\frac{1}{2} k^{2}-\mathrm{i} \delta}+\frac{\Theta(k-1)}{\omega-\frac{1}{2} k^{2}+\mathrm{i} \delta}, \quad \delta \rightarrow 0^{+},
$$

where $G_{0}$ and $G$ are the Green's functions of the ideal Fermi gas and the HEG, respectively. Limiting the summation of the Feynman diagrams for $\Sigma(k, \omega)$ t to those terms that afford correct results for $r_{s} \rightarrow 0$ allows one to apply several rigorous theorems, which yield

(i) the condition for $\mu$ through the Luttinger theorem $\operatorname{Im} \Sigma(1, \mu)=0[15]$,

(ii) the momentum distribution

$$
n(k)=\int \frac{d \omega}{2 \pi \mathrm{i}} \mathrm{e}^{\mathrm{i} \omega \delta} G(k, \omega),
$$

(iii) the quasi-particle weight (through the Luttinger-Ward formula [16])

$$
z_{\mathrm{F}}=\frac{1}{1-\operatorname{Re} \Sigma_{\mathrm{c}}^{\prime}(1, \mu)}, \quad \Sigma_{\mathrm{c}}^{\prime}(k, \omega)=\frac{\partial \Sigma_{\mathrm{c}}(k, \omega)}{\partial \omega},
$$

(iv) the potential energy (through the Galitskii-Migdal formula [17] )

$$
v=\frac{1}{2} \int d\left(k^{3}\right) \int \frac{d \omega}{2 \pi \mathrm{i}} \mathrm{e}^{\mathrm{i} \omega \delta} G(k, \omega) \Sigma(k, \omega) .
$$

Note that $\Sigma=\Sigma_{\mathrm{x}}+\Sigma_{\mathrm{c}}, \Sigma_{\mathrm{c}}=\Sigma_{2}+\Sigma_{3}+\cdots$, and $\Sigma_{2}=\Sigma_{2 \mathrm{~d}}+\Sigma_{2 \mathrm{x}}$. In the lowest order, one has $\Sigma_{\mathrm{x}}=G_{0} v_{0}$ and $v_{\mathrm{x}}=G_{0} \Sigma_{\mathrm{x}}$. With $v_{0}(q)=\alpha r_{s} / q^{2}$ [compare Eq. (A.5)], this produces

$$
\Sigma_{\mathrm{x}}(k)=-\frac{1}{k}\left(1+\frac{1-k^{2}}{2 k} \ln \left|\frac{1+k}{1-k}\right|\right) \frac{\alpha r_{s}}{\pi}, \quad \Sigma_{\mathrm{x}}(1)=-\frac{\alpha r_{s}}{\pi}, \quad v_{\mathrm{x}}=-\frac{3}{4} \frac{\alpha r_{s}}{\pi} .
$$

Note that $\Sigma_{\mathrm{x}}(k)$ does not depend on $\omega$. With $G_{\mathrm{c}}=G-G_{0}$, the correlation part of the potential energy reads

$$
v_{\mathrm{c}}=\left(G_{0}+G_{\mathrm{c}}\right) \Sigma_{\mathrm{c}}+G_{\mathrm{c}} \Sigma_{\mathrm{x}}=G_{0} \Sigma_{\mathrm{c}}+G_{\mathrm{c}}\left(\Sigma_{\mathrm{x}}+\Sigma_{\mathrm{c}}\right)
$$


However, our main interest is the Hugenholtz-van Hove (the Luttinger-Ward) theorem [16, $18]$,

$$
\mu_{\mathrm{c}}=\Sigma_{\mathrm{c}}(1, \mu), \quad \mu=\mu_{0}+\mu_{\mathrm{x}}+\mu_{\mathrm{c}}, \mu_{0}=\frac{1}{2}, \mu_{\mathrm{x}}=-\frac{\alpha r_{s}}{\pi}, \mu_{\mathrm{c}}=\left(\alpha r_{s}\right)^{2} a \ln r_{s}+\cdots
$$

The rhs of the above equation depends on $r_{s}$ through both $\Sigma_{\mathrm{c}}(k, \omega)$ and $\mu$. At the limit of $r_{s} \rightarrow 0, \mu$ can be replaced by $\mu_{0}=1 / 2$.

The ring-diagram summation is equivalent to setting $v_{\mathrm{r}}=v_{0}+v_{0} Q v_{\mathrm{r}}$, where $Q(q, \omega)$ is the polarization propagator [in the lowest order, see Eq. (A.1) in the Appendix]. For the self-energy, this means that $\Sigma^{\mathrm{r}}=G_{0} v_{\mathrm{r}}$. It is easy to show that employing the correlation part $\Sigma_{\mathrm{c}}^{\mathrm{r}}=G_{0}\left(v_{\mathrm{r}}-v_{0}\right)$ of $\Sigma^{\mathrm{r}}$ in conjunction with Eqs. (1.4)and (1.6) results in the RPA approximations for $n_{\mathrm{c}}^{\mathrm{r}}(k)[5,11]$ and $v_{\mathrm{c}}^{\mathrm{r}}[5,13]$, respectively. In this paper, we show that $\Sigma_{\mathrm{c}}^{\mathrm{r}}$ is also the proper rhs for Eqs. (1.5) and (1.9) at the limit of $r_{s} \rightarrow 0$, the remainder $\Sigma_{\mathrm{c}}^{\mathrm{nr}}=\Sigma_{\mathrm{c}}-\Sigma_{\mathrm{c}}^{\mathrm{r}}$ contributing only to the higher-order terms. We also investigate whether $\Sigma_{\mathrm{c}}^{\mathrm{HF}}=\left(G-G_{0}\right) v_{0}$, which appears in ref. [21], is an alternative candidate for the rhs of Eq. (1.9). In this case, we find that the "remainder" $\Sigma_{\mathrm{c}}^{\mathrm{nHF}}=\Sigma_{\mathrm{c}}-\Sigma_{\mathrm{c}}^{\mathrm{HF}}=\Sigma_{\mathrm{c}}^{\mathrm{r}}+\cdots$ determines the lowest-order terms and $\Sigma_{\mathrm{c}}^{\mathrm{HF}}$ contributes only to the higher-order ones.

\section{THE RING-DIAGRAM SELF-ENERGY $\Sigma_{\mathrm{c}}^{\mathrm{r}}(k, \omega)$}

According to the diagram rules, the ring-diagram-summed self-energy is given by

$$
\begin{aligned}
\Sigma_{\mathrm{c}}^{\mathrm{r}}(k, \omega) & =\left(\alpha r_{s}\right)^{2} \frac{2}{\pi^{3}} \int \frac{d^{3} q}{q^{2}} \int \frac{d \eta}{2 \pi \mathrm{i}} \frac{Q(q, \eta)}{q^{2}+q_{c}^{2} Q(q, \eta)} \times \\
& \times\left[\frac{\Theta(|\boldsymbol{k}+\boldsymbol{q}|-1)}{\omega+\eta-\frac{1}{2} k^{2}-\boldsymbol{q} \cdot\left(\boldsymbol{k}+\frac{1}{2} \boldsymbol{q}\right)+\mathrm{i} \delta}+\frac{\Theta(1-|\boldsymbol{k}+\boldsymbol{q}|)}{\omega+\eta-\frac{1}{2} k^{2}-\boldsymbol{q} \cdot\left(\boldsymbol{k}+\frac{1}{2} \boldsymbol{q}\right)-\mathrm{i} \delta}\right]
\end{aligned}
$$

If in the above equation the term $q_{c}^{2} Q(q, \eta)$, which describes the RPA screening of the bare Coulomb repulsion of $\alpha r_{s} / q^{2}$, is deleted, $\Sigma_{\mathrm{c}}^{\mathrm{r}}(k, \omega)$ simplifies to $\Sigma_{2 \mathrm{~d}}(k, \omega)$. Whereas $\Sigma_{2 \mathrm{~d}}=\operatorname{Re} \Sigma_{2 \mathrm{~d}}(1,1 / 2)$ diverges with an artificial cut-off $q_{0}$ according to $\left(\alpha r_{s}\right)^{2} \int_{q_{0}} d q / q$, the ring-diagram sum $\Sigma_{\mathrm{c}}^{\mathrm{r}}=\operatorname{Re} \Sigma_{\mathrm{c}}^{\mathrm{r}}(1,1 / 2)$ is non-divergent, as it effectively replaces $q_{0}$ by the "natural" cut-off $q_{\mathrm{c}} \sim \sqrt{r}_{s}$, producing $\Sigma_{\mathrm{c}}^{\mathrm{r}} \sim\left(\alpha r_{s}\right)^{2} \ln r_{s}$. We follow the procedure of GellMann and Brueckner for the correlation energy [9]. Upon the substitution $\eta=\mathrm{i} q u$ and 
contour deformation from the real to the imaginary axis, one arrives at

$$
\begin{aligned}
\Sigma_{\mathrm{c}}^{\mathrm{r}} & =-\frac{\left(\alpha r_{s}\right)^{2}}{\pi^{4}} \int_{0}^{\infty} d u \int \frac{d^{3} q}{q^{2}} \frac{R(q, u)}{q^{2}+q_{c}^{2} R(q, u)} \cdot \frac{2\left(x+\frac{q}{2}\right)}{u^{2}+\left(x+\frac{q}{2}\right)^{2}} \\
& =-\left(\alpha r_{s}\right)^{2} \frac{2}{\pi^{3}} \int_{0}^{\infty} d u \int_{0}^{\infty} d q \frac{R(q, u)}{q^{2}+q_{c}^{2} R(q, u)} \cdot \ln \frac{u^{2}+\left(\frac{q}{2}+1\right)^{2}}{u^{2}+\left(\frac{q}{2}-1\right)^{2}}
\end{aligned}
$$

The asymptotic behavior for $r_{s} \rightarrow 0$ is determined by the lower integration limit of $q \rightarrow 0$, which allows for the approximate replacements of $R(q, u)$ with $R_{0}(u)$ [setting $R_{0}(u) \neq 0$ makes the Coulomb repulsion effectively screened] and $\ln [\cdots]$ with $2 q /\left(1+u^{2}\right)$ that yields

$$
\Sigma_{\mathrm{c}}^{\mathrm{r}}=\left(\alpha r_{s}\right)^{2}\left[\left(\frac{2}{\pi^{3}} \int_{0}^{\infty} d u \frac{R_{0}(u)}{1+u^{2}}\right) \ln r_{s}+\text { const }+\cdots\right] .
$$

[see Eq. (A.4) in the Appendix for the integral]. The resulting $\Sigma_{\mathrm{c}}^{\mathrm{r}}=\left(\alpha r_{s}\right)^{2}\left(a \ln r_{s}+\right.$ const + ...) is in full agreement with the lhs of the Hugenholtz-van Hove theorem [Eq. (1.9)].

The frequency derivative $\Sigma_{\mathrm{c}}^{\mathrm{r} \prime}=\Sigma_{\mathrm{c}}^{\mathrm{r}}(1,1 / 2)$ can be treated similarly [20],

$$
\begin{aligned}
\Sigma_{\mathrm{c}}^{\mathrm{r} \prime} & =\frac{\left(\alpha r_{s}\right)^{2}}{\pi^{4}} \int \frac{d^{3} q}{q^{3}} \int d u \frac{R(q, u)}{q^{2}+q_{c}^{2} R(q, u)} \frac{\partial}{\partial u} \frac{u}{u^{2}+\left(x+\frac{q}{2}\right)^{2}} \\
& =-\frac{\left(\alpha r_{s}\right)^{2}}{\pi^{4}} \int \frac{d^{3} q}{q^{3}} \int d u \frac{R(q, u)}{q^{2}+q_{c}^{2} R(q, u)} \frac{\partial}{\partial u} \frac{1}{2}\left(\arctan \frac{1+\frac{q}{2}}{u}+\arctan \frac{1-\frac{q}{2}}{u}\right)_{\delta} .
\end{aligned}
$$

Note that a thin layer of vanishing thickness $\delta$ has to be deleted along $|\boldsymbol{e}+\boldsymbol{q}|$, allowing integration by parts. The small- $q$ replacements $R(q, u)$ with $R_{0}(u)$ and $\arctan (1 \pm q / 2) / u$ with $\arctan 1 / u$ yield

$$
\Sigma_{\mathrm{c}}^{\mathrm{r} \prime}=\left(\frac{\alpha}{\pi^{2}} \int_{0}^{\infty} d u \frac{R_{0}^{\prime}(u)}{R_{0}(u)} \arctan \frac{1}{u}\right) r_{s}+O\left(r_{s}^{2}\right)
$$

[see Eq. (A.4) in the Appendix for the integral]. Combining this equation with Eq. (1.5) affords the well known RPA result of $z_{\mathrm{F}}=1 /\left(1-\Sigma_{\mathrm{c}}^{\mathrm{r} \prime}\right)=1+\Sigma_{\mathrm{c}}^{\mathrm{r} \prime}+\cdots=1-0.18 r_{s}+\cdots$ [11]. 


\section{THE HARTEE-FOCK SELF-ENERGY $\Sigma_{\mathrm{c}}^{\mathrm{HF}}(k)$}

Since the bare Coulomb repulsion $v_{0}(q)$ is a static one, the Hartree-Fock self-energy $\Sigma_{\mathrm{c}}^{\mathrm{HF}}=$ $\left(G-G_{0}\right) v_{0}$ is given by the momentum distribution $n(k)$ alone [21]

$$
\Sigma_{\mathrm{c}}^{\mathrm{HF}}(k)=\frac{\alpha r_{s}}{\pi} \frac{1}{k} \int_{0}^{\infty} d k^{\prime} k^{\prime} \ln \left|\frac{k-k^{\prime}}{k+k^{\prime}}\right| n_{\mathrm{c}}\left(k^{\prime}\right), \quad n_{\mathrm{c}}(k)=n(k)-\Theta(1-k) .
$$

In the above equation, the factor in front of $n(k)$ arises from the Coulomb repulsion. Because $\Sigma_{\mathrm{c}}^{\mathrm{HF}}(k)$ does not depend on $\omega$, it cannot contribute to the deviations of $n(k)$ from $\Theta(1-k)$ and of $z_{\mathrm{F}}$ from 1 according to Eqs. (1.4) and (1.5). Such deviations are caused by the non-HF part $\Sigma_{\mathrm{c}}^{\mathrm{nHF}}=\Sigma_{\mathrm{c}}-\Sigma_{\mathrm{c}}^{\mathrm{HF}}=\Sigma_{\mathrm{c}}^{\mathrm{r}}+\cdots$. For $n(k)$ set to $\Theta(1-k)$, the Galitskii-Migdal formula [Eq. (1.6)] yields the lowest-order exchange energy $v_{\mathrm{x}}=-\frac{3}{4} \frac{\alpha r_{s}}{\pi}$, whereas for the actual $n(k)$ it produces the full exchange or Fock energy,

$$
v_{\mathrm{F}}=-\frac{3}{2} \frac{\alpha r_{s}}{\pi} \int_{0}^{\infty} d k \int_{0}^{\infty} d k^{\prime} n(k) n\left(k^{\prime}\right) k k^{\prime} \ln \left|\frac{k+k^{\prime}}{k-k^{\prime}}\right|
$$

which constitutes only one component of the exact potential energy $v$ [see Eq. (43) of ref. [12]]. Consider the (dimensionless) pair density $g(r)$ and its cumulant partitioning $g(r)=1-\frac{1}{2} f^{2}(r)-h(r)[3]$, where $f(r)$ is the (dimensionless) one-body density matrix [i.e. the Fourier transform of $n(k)$ ] and $h(r)$ is the cumulant pair density [i.e. the diagonal part of the cumulant (non-reducible) two-body density matrix]. The potential energy $v=v_{\mathrm{F}}+v_{\text {cum }}$ follows from the full pair density $g(r)$. The Hartree term $g_{0}(r)=1$ is compensated by the positive background, whereas $g_{\mathrm{x}}(r)=-\frac{1}{2} f^{2}(r)$ and $g_{\text {cum }}(r)=-h(r)$ give rise to $v_{\mathrm{F}}$ of Eq. (3.2) and $v_{\text {cum }}$, respectively. Consequently, the knowledge of the non-HF part $\Sigma_{\mathrm{c}}^{\mathrm{nHF}}=\Sigma_{\mathrm{c}}^{\mathrm{r}}+\cdots$ is essential for proper evaluation of $n(k), z_{\mathrm{F}}$, and $v$. One may inquire whether it is nevertheless possible to employ the expression (3.1) in Eq. (1.9). Within perturbation theory, the leading term of $n_{\mathrm{c}}(k)$ is proportional to $r_{s}^{2}$, requiring that $\Sigma_{\mathrm{c}}^{\mathrm{HF}}(1) \sim r_{s}^{3}$, which contradicts the scaling $\mu_{\mathrm{c}} \sim r_{s}^{2}$. The following analysis demonstrates that this contradiction remains after the ring-diagram summation, which turns out to yield, respectively, $r_{s}^{2} \ln r_{s}$ and $r_{s}^{3} \ln r_{s}$ as the leading terms for the lhs and rhs of Eq. (1.9). 
Because of the availability of exact $n_{\mathrm{c}}(k)[11]$, the rhs of

$$
\Sigma_{\mathrm{c}}^{\mathrm{HF}}(1)=\frac{\alpha r_{s}}{\pi} I, I=\int_{0}^{\infty} d k n_{\mathrm{c}}(k) f(k), f(k)=k \ln \left|\frac{1-k}{1+k}\right|
$$

can be readily computed at the weak-correlation limit of $r_{s} \rightarrow 0$. In the following, the approach previously employed in relating the small- $r_{s}$ non-analyticities of $t_{\mathrm{c}}$ and $v_{\mathrm{c}}$ to the peculiarities of $n_{\mathrm{c}}(k)$ and the static structure factor $S_{\mathrm{c}}(q)$ at the limit of $r_{s} \rightarrow 0[5]$ is used.

The small- $r_{s}$ behavior of the rhs of Eq. (3.1) is determined by the behavior of $n_{c}(k)$ near the Fermi surface. As shown by Daniel, Vosko, and Kulik [11], and reiterated in later works $[4,5]$, two functions are needed to describe this behavior, namely $F(k)$ with the properties $F(k \rightarrow 0)=4.11234+O\left(k^{2}\right), \quad F(k \rightarrow \infty)=\frac{8 \pi^{2}}{9} \frac{1}{k^{8}}+O\left(\frac{1}{k^{10}}\right), \quad F(k \rightarrow 1)=\frac{\pi^{2}}{3} \frac{1-\ln 2}{k^{2}(1-k)^{2}}$.

and $G(x)$ with the asymptotics

$$
G(0)=3.35334, \quad G(x \gg 1)=\frac{\pi}{6} \frac{1-\ln 2}{x^{2}}+O\left(\frac{1}{x^{4}}\right) .
$$

Near $k=1$, the function $n_{c}(k)$ is given by

$$
n_{\mathrm{c}}(k)=\left(\frac{q_{\mathrm{c}}^{2}}{4 \pi}\right)^{2} \cdot \begin{cases}-F(k), & 0<k<1-\xi \\ -\frac{2 \pi}{q_{c}^{2}} \frac{1}{k^{2}} G\left(\frac{1-k}{q_{c}}\right), & 1-\xi<k<1 \\ +\frac{2 \pi}{q_{c}^{2}} \frac{1}{k^{2}} G\left(\frac{k-1}{q_{c}}\right), & 1<k<1+\xi \\ +F(k), & 1+\xi<k\end{cases}
$$

where $1 \gg \xi \gg q_{c}[5]$. The function $F(k)$ contributes to $I=I_{F}+I_{G}$ through the expression

$$
\begin{aligned}
& I_{F}=I_{F}^{>}+I_{F}^{<} \\
& I_{F}^{>}=\left(\frac{q_{c}^{2}}{4 \pi}\right)^{2} \int_{1+\xi}^{\infty} d k F(k) f(k), \quad I_{F}^{<}=-\left(\frac{q_{c}^{2}}{4 \pi}\right)^{2} \int_{0}^{1-\xi} d k F(k) f(k) .
\end{aligned}
$$

With a fixed positive number $A$ sufficiently small to assure that $F(k)$ can be replaced by its asymptotics (3.4), one obtains

$$
\begin{aligned}
& I_{F}^{>} \approx\left(\frac{q_{c}^{2}}{4 \pi}\right)^{2}\left[\int_{1+A}^{\infty} d k F(k) f(k)+\frac{\pi^{2}}{3}(1-\ln 2) \int_{1+\xi}^{1+A} d k \frac{f(k)}{k^{2}(1-k)^{2}}\right] \\
& =\quad O\left(r_{s}^{2}\right) \quad+q_{c}^{4} \frac{1-\ln 2}{48} \int_{\xi}^{A} d k \frac{f(1+k)}{(1+k)^{2} k^{2}} .
\end{aligned}
$$


The result for $I_{F}^{<}$is similar, the above integrand being replaced by $-\frac{f(1-k)}{(1-k)^{2} k^{2}}$. Therefore

$$
I_{F} \approx O\left(r_{s}^{2}\right)+q_{c}^{4} \frac{1-\ln 2}{48} \int_{\xi}^{A} \frac{d k}{k^{2}} w(k), \quad w(k)=\frac{f(1+k)}{(1+k)^{2}}-\frac{f(1-k)}{(1-k)^{2}} .
$$

The contribution of $G(x)$ to $I=I_{F}+I_{G}$ is treated analogously,

$$
\begin{gathered}
I_{G}=I_{G}^{>}+I_{G}^{<} \\
I_{G}^{>}=\frac{q_{c}^{2}}{8 \pi} \int_{1}^{1+\xi} d k \frac{1}{k^{2}} G\left(\frac{|k-1|}{q_{c}}\right) f(k) \quad, \quad I_{G}^{<}=-\frac{q_{c}^{2}}{8 \pi} \int_{1-\xi}^{1} d k \frac{1}{k^{2}} G\left(\frac{|k-1|}{q_{c}}\right) f(k) .
\end{gathered}
$$

With a fixed positive number $B$ sufficiently large to assure that $G(x)$ can be replaced by its asymptotics (3.5), it follows that

$$
\begin{array}{r}
I_{G}^{>}=\frac{q_{c}^{2}}{8 \pi}\left[\int_{1}^{1+q_{c} B}+\int_{1+q_{c} B}^{1+\xi}\right] \frac{d k}{k^{2}} G\left(\frac{|k-1|}{q_{c}}\right) f(k) \\
\approx \frac{q_{c}^{3}}{8 \pi} \int_{0}^{B} d x G(x) \frac{f\left(1+q_{c} x\right)}{\left(1+q_{c} x\right)^{2}}+q_{c}^{4} \frac{1-\ln 2}{48} \int_{q_{c} B}^{\xi} \frac{d k}{k^{2}} \frac{f(1+k)}{(1+k)^{2}} .
\end{array}
$$

The result for $I_{G}^{<}$is similar, the respective parts of the first and second integrands being replaced by $-\frac{f\left(1-q_{c} x\right)}{\left(1-q_{c} x\right)^{2}}$ and $-\frac{f(1-k)}{(1-k)^{2}}$. Therefore,

$$
I_{G} \approx \frac{q_{c}^{3}}{8 \pi} \int_{0}^{B} d x G(x) w\left(q_{c} x\right)+q_{c}^{4} \frac{1-\ln 2}{48} \int_{q_{c} B}^{\xi} \frac{d k}{k^{2}} w(k) .
$$

Combining the above estimates, one obtains

$$
I \approx O\left(r_{s}^{2}\right)+\frac{q_{c}^{3}}{8 \pi} \int_{0}^{B} d x G(x) w\left(q_{c} x\right)+q_{c}^{4} \frac{1-\ln 2}{48} \int_{q_{c} B}^{A} \frac{d k}{k^{2}} w(k) .
$$

Since for a sufficiently small positive $k$

$$
w(k)=\frac{1}{1+k} \ln \left|\frac{k}{2+k}\right|-\frac{1}{1-k} \ln \left|\frac{k}{1-k}\right| \approx-2 k \ln k,
$$

the integrals of Eq. (3.13) yield the leading terms of

$$
\frac{q_{c}^{3}}{8 \pi} \int_{0}^{B} d x G(x)\left(-2 q_{c} x\right) \ln \left(q_{c} x\right)=-q_{c}^{4} \frac{1-\ln 2}{24}\left[\ln q_{c} \ln B+C_{0} \ln q_{c}+\frac{1}{2}(\ln B)^{2}\right],
$$

where the constant $C_{0}$ does not depend on $\mathrm{B}$, and

$$
q_{c}^{4} \frac{1-\ln 2}{48} \int_{q_{c} B}^{A} \frac{d k}{k^{2}}(-2 k) \ln k=q_{c}^{4} \frac{1-\ln 2}{48}\left[\left(\ln q_{c}+\ln B\right)^{2}-(\ln A)^{2}\right]
$$

(note that cancellation of the terms dependent on $B$ in the combined integrals). Thus $\Sigma_{c}^{\mathrm{HF}}(1)=\left(\frac{\alpha r_{s}}{\pi}\right)^{3} \frac{1-\ln 2}{12}\left[\left(\ln r_{s}\right)^{2}-2 C_{0} \ln r_{s}\right]+\cdots$, which clearly demonstrates that for $r_{s} \rightarrow 0$ the non-HF term $\Sigma_{\mathrm{c}}^{\mathrm{nHF}}(1,1 / 2)=\Sigma_{\mathrm{c}}^{\mathrm{r}}(1,1 / 2)+\cdots$ has to be used in the rhs of Eq. (1.9). In summary, the terms that correctly describe the small- $r_{s}$ behavior are contained in $\Sigma_{\mathrm{c}}^{\mathrm{nHF}}(k, \omega)=\Sigma_{\mathrm{c}}^{\mathrm{r}}(k, \omega)+\cdots[22]$. However, $\Sigma_{\mathrm{c}}^{\mathrm{HF}}(1)$, together with $v_{\mathrm{F}}-v_{\mathrm{x}}$, can serve as measures of the correlation strength, see refs. [12] and [23]. 


\section{CONCLUSIONS}

The correct small- $r_{s}$ behavior of the correlation contribution $\Sigma_{\mathrm{c}}(k, \omega)$ to the self-energy is given by the ring-diagram-summed $\Sigma_{\mathrm{c}}^{\mathrm{r}}(k, \omega)$. The summation eliminates the divergence of $\Sigma_{2 \mathrm{~d}}(1,1 / 2) \sim r_{s}^{2} \int_{0} d q / q$ and of $n_{2 \mathrm{~d}}(k)$ at the Fermi surface. Upon application of the Galitskii-Midgal formula, the correct potential energy $v_{\mathrm{c}}=2 a\left(\alpha r_{s}\right)^{2} \ln r_{s}+\cdots$ results. The derivative $\partial \Sigma_{\mathrm{c}}^{\mathrm{r}}(1, \omega) /\left.\partial \omega\right|_{\omega=1 / 2}$ used in conjunction with the Luttinger-Ward formula affords the correct $z_{\mathrm{F}}=1-0.18 r_{s}+\cdots$ for $r_{s} \rightarrow 0$. Finally, $\Sigma_{\mathrm{c}}^{\mathrm{r}}(1,1 / 2)=\left(\alpha r_{s}\right)^{2}\left(a \ln r_{s}+\right.$ const $\left.+\cdots\right)$ is in full agreement with the Hugenholtz-van Hove formula $\mu_{\mathrm{c}}=\Sigma_{\mathrm{c}}(1, \mu)$ with $\mu \rightarrow 1 / 2$ at the limit of $r_{s} \rightarrow 0$.

\section{Ackowledgment}

The research described in this publication has been supported by Komitet Badan Naukowych under the grant 4 T09A 031 25. One of the authors (P.Z.) acknowledges P. Fulde for support of this work and the other (J.C.) extends his thanks to MPI PKS Dresden for hospitality and research fellowship.

[1] M.P. Tosi in: N.H. March (Ed.), Electron Correlation in the Solid State, Imperial College Press, 1999, p. 1.

[2] There is a non-vanishing probability of finding no electron, as well as 2, 3, or more electrons in such a Wigner sphere. Such particle number fluctuations in fragments have been studied by P. Ziesche, J. Tao, M. Seidl, and J.P. Perdew, Int. J. Quantum Chem. 77, 819 (2000), resulting in the conclusion that "correlation suppresses fluctuations", cf. also P. Fulde, Electron Correlations in Molecules and Solids, 3rd ed., Springer, Berlin, 1995, p.157.

[3] P. Ziesche, Int J. Quantum Chem. 90, 342 (2002).

[4] J. Cioslowski, P. Ziesche, and K. Pernal, Phys. Rev. B 63, 205105 (2001); J. Chem. Phys. 115, 8725 (2001).

[5] P. Ziesche and J. Cioslowski, Physica A 356, 598 (2005). This paper elucidates the peculiarities of $n(k)$ and $S(q)$ inherent in the RPA lead to $e_{\mathrm{c}}=a\left(\alpha r_{s}\right)^{2} \ln r_{s}$.

[6] R.D. Muiño, I. Nagy, and P.M. Echenique, Phys. Rev. B 72, 075117 (2005). 
[7] W. Heisenberg, Z. Naturf. 2a, 185 (1947).

[8] W. Macke, Z. Naturf. 5a, 192 (1950).

[9] M. Gell-Mann and K. Brueckner, Phys. Rev. 106, 364 (1957).

[10] N.H. March, Phys. Rev. 110, 604 (1958).

[11] E. Daniel and S.H. Vosko, Phys. Rev. 120, 2041 (1960); I.O. Kulik, Zh. Éksp. Teor. Fiz. 40, 1343 (1961) [Sov. Phys. JETP 13, 946 (1961)]. The intention of the work reported in ref. [12] was to construct an approximate expression for $n\left(k, r_{s}\right)$ valid for values of $r_{s}$ between 1 and 10. The fitted expressions quoted in that paper are not accurate enough to fully recover the well-known RPA behavior for $r_{s} \rightarrow 0^{+}$. In ref. [4], two functions $F(k)$ and $G(x)$ are used to describe the peculiar behavior of $n(k)$ near the Fermi surface.

[12] P. Gori-Giorgi and P. Ziesche, Phys. Rev. B 66, 235116 (2002).

[13] J.C. Kimball, Phys. Rev. B 14, 2371 (1976).

[14] F. Seitz, Modern Theory of Solids, McGraw-Hill, New York, 1940, Sec. 76.

[15] J.M. Luttinger, Phys. Rev. 121, 942, 1251 (1961).

[16] J.M. Luttinger and J.C. Ward, Phys. Rev. 118, 1417 (1960).

[17] W.M. Galitskii and A.B. Migdal, Zh. Éksp. Teor. Fiz. 34, 139 (1958) [Sov. Phys. JETP 7, 96 (1958)].

[18] N.M. Hugenholtz and L. van Hove, Physica 24, 363 (1958). Their theorem has been brought into the form $\mu-\mu_{0}=\Sigma(1, \mu)$ in ref. [16]. In ref. [19], an alternative formulation is provided as "a highly suggestive form".

[19] A. Klein and R. Prange, Phys. Rev. 112, 994 (1958).

[20] P. Ziesche, Thesis, TU Dresden, 1963; Ann. Physik (Leipzig) 21, 80 (1968).

[21] B. Farid, Phil. Mag. 84, 109 (2004).

[22] In particular we conclude that $\Sigma_{\mathrm{c}}^{\mathrm{nHF}}(1, \mu) \neq 0$ (otherwise than in [21]).

[23] A.D. Gottlieb and N.J. Mauser, Phys. Rev. Lett. 95, 123003 (2005).

\section{APPENDIX A: THE POLARIZATION PROPAGATOR}

The polarization propagator (in the lowest order) is given by

$$
Q(q, \eta)=\int \frac{d^{3} k}{4 \pi}\left[\frac{1}{\boldsymbol{q}\left(\boldsymbol{k}+\frac{1}{2} \boldsymbol{q}\right)-\eta-\mathrm{i} \delta}+\frac{1}{\boldsymbol{q}\left(\boldsymbol{k}+\frac{1}{2} \boldsymbol{q}\right)+\eta-\mathrm{i} \delta}\right] \Theta(1-k) \Theta(|\boldsymbol{k}+\boldsymbol{q}|-1) .
$$


For $\eta=\mathrm{i} q u$, a real function of $q$ and $u$ arises [11],

$$
\begin{aligned}
R(q, u)=Q(q, \mathrm{i} q u) & =\frac{1}{2}\left[1+\frac{1+u^{2}-\frac{q^{2}}{4}}{2 q} \ln \frac{\left(\frac{q}{2}+1\right)^{2}+u^{2}}{\left(\frac{q}{2}-1\right)^{2}+u^{2}}\right. \\
& \left.-u\left(\arctan \frac{1+\frac{q}{2}}{u}+\arctan \frac{1-\frac{q}{2}}{u}\right)\right]
\end{aligned}
$$

which is even in $u$. The function $R(q, u)$ has the small- $q$ expansion $R(q, u)=R_{0}(u)+O\left(q^{2}\right)$ with

$$
R_{0}(u)=1-u \arctan \frac{1}{u}
$$

The integrals

$$
\int_{0}^{\infty} d u \frac{R_{0}(u)}{1+u^{2}}=\frac{\pi}{2}(1-\ln 2) \approx 0.482003 \quad \text { and } \quad \int_{0}^{\infty} d u \frac{R_{0}^{\prime}(u)}{R_{0}(u)} \arctan \frac{1}{u} \approx-3.353337
$$

appear in section II of this paper. The integrals

$$
\int_{0}^{1} d k^{\prime} k^{\prime} \ln \left|\frac{k+k^{\prime}}{k-k^{\prime}}\right|=1+\frac{1-k^{2}}{2 k} \ln \left|\frac{1+k}{1-k}\right| \quad \text { and } \quad \int_{0}^{1} d k \int_{0}^{1} d k^{\prime} k k^{\prime} \ln \left|\frac{k+k^{\prime}}{k-k^{\prime}}\right|=\frac{1}{2}
$$

appear in sections I and III. 